\title{
Comportamento tribocorrosivo de sistemas metalocerâmicos odontológicos de Ni-Cr/Porcelana e Ni-Cr/Ti/Porcelana
}

\section{(Tribocorrosive behavior of odontological Ni-Cr/Porcelain and Ni-Cr/Ti/Porcelain metal-ceramic systems)}

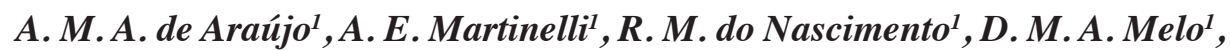 \\ A. L. Medeiros ${ }^{1}$, L.A.S.M da Rocha ${ }^{2}$, E.A. Ávila ${ }^{2}$ \\ ${ }^{1}$ Universidade Federal do Rio Grande do Norte, Av. Sen. S. Filho 3000, Campus Lagoa Nova, Natal, RN 59078-970 \\ ${ }^{2}$ Departamento de Engenharia Mecânica, Universidade do Minho, Portugal \\ dartarios@yahoo.com.br,aemart@uol.com.br,dmelo@matrix.com.br,almedeiros@hotmail.com.br, \\ daraujomelo@gmail.com,valeska@eq.ufrn.br
}

\begin{abstract}
Resumo
Próteses parciais fixas são empregadas na reabilitação da forma e função de dentes parcial ou totalmente comprometidos e devem permanecer permanentemente unidas aos remanescentes dentários. O material mais utilizado na confecção de próteses odontológicas é a porcelana feldspática. Contudo, ela apresenta propriedades mecânicas limitadas para reposição de espaços edêntulos extensos. A associação da porcelana com sistemas metálicos de níquel-cromo (sistema metalocerâmico) permite que a infra-estrutura metálica compense a natureza frágil da porcelana, preservando-se tanto a isolação térmica como a estética desejáveis, além de reduzir a possibilidade do surgimento e crescimento de trincas durante os esforços mastigatórios. Falhas coesivas por baixa resistência mecânica da porcelana comunicam a estrutura metálica com o ambiente oral, caracterizado pela presença de uma solução eletrolítica (saliva), pela agressividade das variações cíclicas de temperatura, pH e por solicitações mecânicas. Este processo resulta na liberação de íons metálicos que podem desencadear respostas alérgicas ou inflamatórias, e/ ou na degradação clínica precoce do sistema metalocerâmico. Este trabalho teve por objetivo avaliar o efeito da presença de uma camada intermediária de titânio sobre o comportamento de sistemas metalocerâmicos dentários. A aplicação por plasma de um filme fino de titânio gera uma camada óxida passivante regular que protege o substrato metálico contra os efeitos corrosivos da saliva. Ensaios tribocorrosivos foram realizados para simular o ambiente oral, detectando precocemente a formação de trincas no sistema metalocerâmico, o que qualifica a aderência entre os componentes do referido sistema. Foram produzidas amostras planas nas quais suspensões de porcelana feldspática odontológica foram depositadas e queimadas sobre substratos metálicos modificados por filme fino de titânio, comparando-as com o sistema metalocerâmico convencional, sem titânio. O sistema modificado por titânio apresentou melhor comportamento, caracterizando-se pelo menor número de traços de fratura superficial adjacentes à área do ensaio tribocorrosivo.

Palavras-chave: metalocerâmica, tribocorrosão, titânio, metalização.
\end{abstract}

\begin{abstract}
Partial fixed prosthodontic restoration is used to rehabilitate both the form and function of partial or total compromised teeth and must remain permanently joined to the remaining teeth. The most useful material in prosthodontics is feldspar porcelain, commercialized as aluminosilicate powders. Dental porcelains depict limited mechanical strength to rehabilitate extensive spaces. The association of porcelain with $\mathrm{Ni}$-Cr metallic infrastructures allows the metallic component to compensate for the brittle nature of porcelain, thus preserving the thermal insulation, aesthetics and, most important, reducing the possibility of cracking during mastication efforts. Cohesive flaws caused by low mechanical strength connect the metallic substructure to the oral environment, characterized by the presence of an electrolytic solution (saliva), aggressive temperature and pH cycles as well as mechanical demand. As a result, ions are released and can promote allergic or inflammatory responses, in addition to clinical degradation of the prosthodontic system. Therefore, the aim of this study was to evaluate the presence of an intermediate titanium layer on the behavior of $\mathrm{Ni}$-Cr/porcelain systems. Plasma deposition of titanium films were also carried out to create barrier layers capable of isolating the metallic substrate from the hazardous effects of the oral environment. Tribocorrosion tests were performed to simulate the oral environment and corresponding wear, making it possible to early detect crack formation and growth on metal-ceramic systems and estimate the adherence between the metal and ceramic components. Plain samples consisting of dental feldspar porcelain deposited onto metallic substrates coated or not with titanium were fired and characterized. The results showed that the deposition of titanium films improved the adherence of the system compared to conventional metal-ceramic systems, thus holding crack growth towards the metallic component.
\end{abstract}

Keywords: metal-ceramic, tribocorrosion, titanium, metallization. 


\section{INTRODUÇÃO}

Reabilitar dentes perdidos implica na recuperação harmônica da forma, função e estética que se enquadrem em padrões subjetivos e individuais de beleza [1]. As próteses parciais fixas reabilitam dentes vitais ou não comprometidos parcial ou totalmente. Elas devem manter-se unidas permanentemente ao(s) remanescente(s) dentário(s) na substituição de um ou mais elementos ausentes [2]. Dentre as opções comercialmente disponíveis, as ligas de fundição à base de níquel-cromo são as mais utilizadas na confecção de próteses metalocerâmicas [3-7]. Uma vantagem mecânica das ligas de níquel-cromo sobre as ligas áuricas é o módulo de elasticidade, aproximadamente duas vezes superior, traduzido em menor espessura metálica necessária ao suporte da porcelana [8] e, portanto, maior preservação do remanescente dentário no preparo do elemento suporte. Estas ligas apresentam coeficiente de expansão térmica (CET) semelhante ao das porcelanas utilizadas nas próteses metalocerâmicas, prevenindo a tendência à fratura da porcelana durante $\mathrm{o}$ aquecimento e resfriamento do processo de cocção em laboratório $[3,8]$.

Porcelanas associadas às ligas metálicas compõem sistemas metalocerâmicos com uniões formadas com materiais físicos e mecanicamente distintos [9-12]; compensando a natureza frágil da camada de porcelana $[10,13]$ e reduzindo a possibilidade de desenvolvimento de trincas interfaciais durante os esforços mastigatórios. Estes sistemas podem ser empregados na confecção de coroas unitárias e em pontes fixas para espaços edêntulos curtos ou extensos, na região anterior e/ou posterior das arcadas dentárias. O sucesso do sistema metalocerâmico depende especialmente da estabilidade da união [10,11], que ocorre por microrretenção mecânica, ligações tipo van der Waals e, especialmente, pela interação química da camada superficial de óxidos metálicos com a porcelana $[2,5,11,14,15]$. Os óxidos da superfície metálica são indispensáveis à aderência [7, 16, 17]. A formação de uma camada muito fina de óxidos é freqüentemente associada ao aumento dos riscos de insucesso na união metalocerâmica $[11,18]$.

Atualmente há uma tendência em recobrir ligas metálicas de baixo custo por filmes finos estáveis e protetores [19, 20]. Para sistemas metalocerâmicos, o titânio é uma opção biocompatível, quimicamente interessante contra a instabilidade dos óxidos da interface em meio corrosivo e uma alternativa aplicável aos pacientes alérgicos ao níquel. A deposição de filmes em superfícies metálicas pode ser feita por uma variedade de métodos, entre eles, a deposição mecânica e o sputtering. Este último pode ser aprimorado para aumentar o rendimento e a qualidade dos filmes, adicionando-se imãs próximos ao alvo metálico. Com isso, modifica-se a trajetória dos elétrons [21], confinando o plasma na região próxima ao alvo e, portanto, melhorando a eficiência da deposição de filmes finos [22]. Este sistema é denominado triodo magnetron sputtering e torna possível a uniformização do campo elétrico, culminando com maior poder de ionização do alvo, além de viabilizar a produção de filmes com temperatura do substrato inferior a $100{ }^{\circ} \mathrm{C}[23]$.

A boca é um ambiente úmido com oscilações freqüentes de $\mathrm{pH}$ salivar, tornando-se um sistema condutivo ideal para o desenvolvimento de processos corrosivos, que são igualmente influenciados pela variação cíclica dos esforços mastigatórios e das solicitações térmicas, decorrentes da alimentação e/ou de hábitos parafuncionais do sistema estomatognático. A degradação combinada por corrosão e desgaste mecânico em materiais submersos em saliva, solução eletrolítica condutora, pode ser investigada por ensaios tribocorrosivos. A tribocorrosão é um processo químico-eletroquímico-mecânico que leva à degradação de materiais em contato por deslizamento ou rolamento, em ambiente corrosivo. Os testes de tribocorrosão simulam a agressividade e as cargas mastigatórias realizadas na boca, com parâmetros estáticos.

Nesse contexto, este estudo tem por meta avaliar o desempenho de sistemas metalocerâmicos contendo camadas interfaciais de titânio por meio de ensaios tribocorrosivos que simulam cargas mastigatórias em ambiente oral. Para tal foram confeccionados corpos de prova contendo uniões $\mathrm{Ni}-\mathrm{Cr} /$ Porcelana e $\mathrm{Ni}-\mathrm{Cr} / \mathrm{Ti} /$ Porcelana. Os filmes de titânio foram depositados sobre o componente metálico do sistema por dois métodos: mecânico e por plasma. As amostras foram caracterizadas pela técnica da tribocorrosão em ambiente artificial ácido (solução Fusayama) a fim de identificar a formação e propagação de trincas nos sistemas metalocerâmicos. Foi realizada uma avaliação comparativa do comportamento dos sistemas Ni-Cr/porcelana e Ni-Cr/ Ti/Porcelana em função da técnica de deposição de titânio empregada.

\section{MATERIAIS E MÉTODOS}

Uma liga de níquel-cromo (Suprem Cast SB, Talladium do Brasil) para restaurações odontológicas metalocerâmicas foi empregada na confecção dos corpos de prova. A composição química da liga (p.\%) é $61 \% \mathrm{Ni}, 25 \% \mathrm{Cr}$, $10,5 \%$ Mo, $1,5 \%$ Si e $<1 \%$ Ti. Uma porcelana feldspática odontológica (Ceramco III, Dentsply) de baixa fusão $\left(940{ }^{\circ} \mathrm{C}\right)$ foi utilizada no recobrimento da infra-estrutura metálica fundida. A apresentação desta porcelana é sob a forma de pó, contido em frasco plástico opaco. Foram produzidos oito corpos de prova metalocerâmicos, com formato retangular plano de $20,0 \mathrm{~mm}$ x $30,0 \mathrm{~mm}$ x $1,5 \mathrm{~mm}$. Os corpos de prova foram confeccionados em laboratório de prótese dentária, segundo as normas dos fabricantes e divididos em três grupos, em função do tratamento da superfície metálica: CPA: superfície oxidada; CPB: metalização mecânica de Ti; CE: metalização em plasma de Ti.

A oxidação prévia da superfície metálica de contato com a porcelana é preconizada pelo fabricante e conseguida por meio de tratamento térmico. Os corpos de prova submetidos ao processo de oxidação foram inseridos em forno para porcelanas (Phoenix Quick Cool, Ceramco) com temperatura inicial de $650{ }^{\circ} \mathrm{C}$ e pressão de $2,54 \mathrm{cmHg}$, variando $32^{\circ} \mathrm{C}$ por minuto até atingir a temperatura final de $980^{\circ} \mathrm{C}$. Ao término 
do tratamento térmico, o vácuo foi liberado e as amostras gradativamente resfriadas dentro do forno. $\mathrm{O}$ jateamento abrasivo com $\mathrm{Al}_{2} \mathrm{O}_{3}$ em pó $(250 \mu \mathrm{m}$, malha \#60) foi realizado a uma pressão de $65 \mathrm{kgf} / \mathrm{cm}^{2}$, compatível com a malha, para remover impurezas na superfície, reduzir a camada de óxidos - quando realizado após oxidação - uniformizando a textura superficial. Todas as peças foram novamente limpas em álcool isopropílico por vibração ultrassônica ao término das seções de acabamento de superfície para remoção de possíveis partículas contaminantes impregnadas.

As amostras do grupo CPB foram metalizadas mecanicamente com titânio. Uma ponta cônica de titânio comercialmente puro foi colocada em uma retificadora portátil (Dremel, Multipro) de alta rotação, que funcionou na velocidade de $33.000 \mathrm{rpm}$. A angulação de contato entre a ponta e o corpo de prova foi de $45^{\circ} \mathrm{C}$. Somente a ponta do cone percorreu a superfície metálica. As amostras do grupo CE receberam filme de titânio pelo sistema triodo magnetron sputtering. A deposição foi feita durante $45 \mathrm{~min}$, com pressão do gás de trabalho $\left(50 \% \mathrm{~N}_{2}, 50 \%\right.$ Ar) de $4,0 \mu$ Torr, tensão de $-500 \mathrm{~V}$ sobre o alvo, corrente de 1,0 A no alvo, polarização do substrato de $-30 \mathrm{~V}, 200^{\circ} \mathrm{C}$ de temperatura do substrato e razão aproximada de deposição de titânio sobre o substrato de 0,15 $\mu \mathrm{m} / \mathrm{min}$. Para ambos os tipos de metalização propostos foram obtidas imagens por mapeamento de raios $\mathrm{X}$ por energia dispersiva do filme de titânio depositado, para observar-se a uniformidade do processo.

O processo de aplicação da porcelana é manual, porém, criterioso. Ao pó da porcelana foi adicionada água destilada, misturando-os até a obtenção de uma pasta de porcelana opaca, aplicada sobre a superfície tratada da infra-estrutura metálica com o auxílio de um pincel de uso odontológico. O conjunto foi levado ao forno para o primeiro ciclo de cocção da porcelana, segundo as normas do fabricante. A temperatura final para a porcelana Ceramco III é de $940^{\circ} \mathrm{C}$. Após o completo resfriamento da peça, uma segunda camada de porcelana opaca, mais viscosa, foi aplicada com espessura aproximada de 0,2 $\mathrm{mm}$ nas mesmas condições da primeira camada. O conjunto retornou ao forno para o segundo ciclo de cocção da porcelana. Ao término do resfriamento, a camada de porcelana de corpo foi aplicada, com espessura aproximada de $0,7 \mathrm{~mm}$, em toda a extensão da superfície trabalhada. O processo foi idêntico ao da aplicação da camada anterior. A espessura média final dos corpos de prova ficou em torno de $1,2 \mathrm{~mm}$.

Todas as condições de interface propostas para uniões metalocerâmicas foram submetidas a ensaios de tribocorrosão para avaliar a degradação do material promovida por interações mecânicas em solução eletrolítica. Monitoraram-se as variações do coeficiente de atrito e da corrente de corrosão das amostras. A área exposta à saliva artificial (solução de Fusayama-Meyer, Tabela I) foi de 2,54 $\mathrm{cm}^{2}$. O potencial de corrosão $\left(\mathrm{E}_{\text {corr }}\right)$ dos corpos de prova (potencial em circuito aberto) foi verificado durante $60 \mathrm{~min}$ e em seguida foi aplicada uma carga de $159,9 \mathrm{~N}$ para o início do desgaste. O desgaste mecânico ocorreu por deslizamento recíproco em configuração pino-placa, de um pino tronco- cônico de alumina contra os corpos de prova, ou seja, alumina - sistema metalocerâmico. A amplitude dos ensaios foi de 3,0 $\mathrm{mm}$ e estes aconteceram até que o pino atingisse o substrato metálico. O sistema eletrolítico foi constituído por uma célula com arranjo standard de três eletrodos. Como eletrodo auxiliar foi utilizado um eletrodo de platina; como eletrodo de referência, um eletrodo saturado de calomelano e os eletrodos de trabalho foram formados pelas amostras metalocerâmicas.

Tabela I - Composição química da solução de FusayamaMeyer.

[Table I - Chemical composition of Fusayama-Meyer solution.]

\begin{tabular}{|c|c|}
\hline Eletrólito & Concentração (g/L) \\
\hline$\overline{\mathrm{KCl}}$ & $\overline{0,4}$ \\
\hline $\mathrm{NaCl}$ & 0,4 \\
\hline $\mathrm{CaCl}_{2} \cdot 2 \mathrm{H}_{2} \mathrm{O}$ & 0,906 \\
\hline $\mathrm{NaH}_{2} \mathrm{PO}_{4} \cdot 2 \mathrm{H}_{2} \mathrm{O}$ & 0,69 \\
\hline $\mathrm{Na}_{2} \mathrm{~S} .9 \mathrm{H}_{2} \mathrm{O}$ & 0,005 \\
\hline Uréia & 1,0 \\
\hline
\end{tabular}

Os corpos de prova foram embutidos em resina de cura rápida e seccionados transversalmente com disco de diamante em alta velocidade em uma máquina de corte ISOMET (Buehler). O lixamento deu-se por intermédio de uma lixadeira mecânica rotativa (APL-40; Arotec) com lixas de $\mathrm{SiC}$ em granulação decrescente $(320,400,600$ e 1200 grãos/pol ${ }^{2}$ ), polidos na mesma máquina com disco de feltro embebido em pasta de $\mathrm{Al}_{2} \mathrm{O}_{3}(3 \mu \mathrm{m}, 1 \mu \mathrm{m}$ e $0,5 \mu \mathrm{m})$. Os corpos de prova foram metalizados com filme fino de ouro para inspeção em microscópio eletrônico de varredura. A caracterização microestrutural e dos padrões de fratura da liga metálica, da interface e das porcelanas foi realizada em microscópio (Shimadzu SSX-550), acoplado a um sistema de deteç̧ão de raios X por EDS para a determinação da composição qualitativa e semi-quantitativa dos corpos de prova, além da geração de mapas elementares a partir da emissão de raios $\mathrm{X}$ característicos.

\section{RESULTADOS E DISCUSSÃO}

As superfícies da liga Ni-Cr modificadas pela deposição mecânica ou em plasma de titânio foram avaliadas por mapeamento de raios X (Fig. 1). A técnica permite comparar a uniformidade dos filmes de titânio depositados. Na região metalizada mecanicamente (Fig. 1a), observa-se uma descontinuidade significativa no filme de titânio, localizado acima da linha externa tracejada. Isto interfere na qualidade da camada óxida modificada na interface de união do sistema. Já a superfície metalizada em plasma (Fig. 1b) exibe um filme contínuo de titânio, disposto na porção superior da figura, sugerindo uniformidade na interação. A alta energia dos átomos produzidos no sistema triodo magnetron sputtering resulta em filmes compactos e, normalmente mais finos que os depositados mecanicamente. 

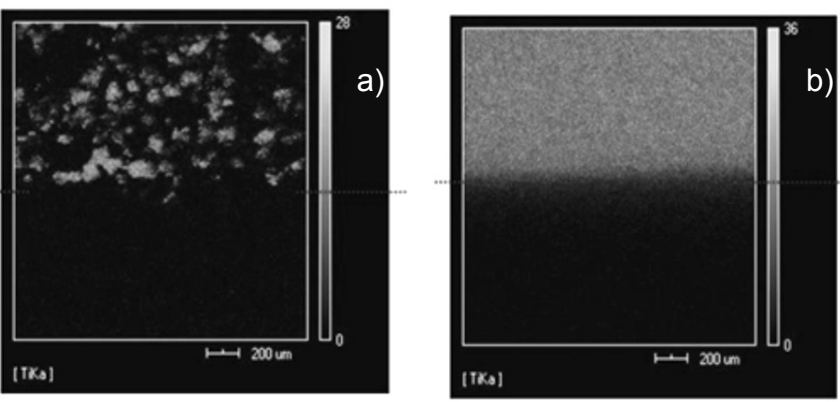

Figura 1: Mapeamento por EDS de superfícies da liga de $\mathrm{Ni}-\mathrm{Cr}$ a) metalizada mecanicamente e b) metalização em triodo magnetron sputtering.

[Figure 1: EDS mapping of $\mathrm{Ni}$-Cr surface after a) mechanical metallization and b) plasma metallization.]

Foram realizados ensaios tribocorrosivos dos sistemas metalocerâmicos visando avaliar a formação e o crescimento de trincas no material cerâmico em condições simuladas de esforços mastigatórios, sem impacto, em ambiente oral e a aderência da união metalocerâmica. Os ensaios tribocorrosivos evidenciaram um aumento do coeficiente de atrito no início do ensaio, correspondente ao período de rodagem, ou contato inicial do pino de alumina contra a superfície de porcelana. Após um determinado tempo de deslizamento, característico de cada amostra, a fratura final coincide com o aumento brusco do coeficiente de atrito e da corrente de corrosão, finalizando com a queda repentina desses dois parâmetros.

Para amostra do grupo CPA (jateada e sem intercamada de titânio), estes eventos ocorreram após $225 \mathrm{~min}$ de ensaio. Uma análise mais detalhada do comportamento tribocorrosivo dessa amostra (Fig. 2), com aumento na escala de valores de corrente, ilustra a capacidade da técnica em detectar a formação de trincas. Após 125 min de ensaio, notou-se um aumento nos valores de corrente, contudo sem que isso representasse mudanças em nível de atrito. Portanto,

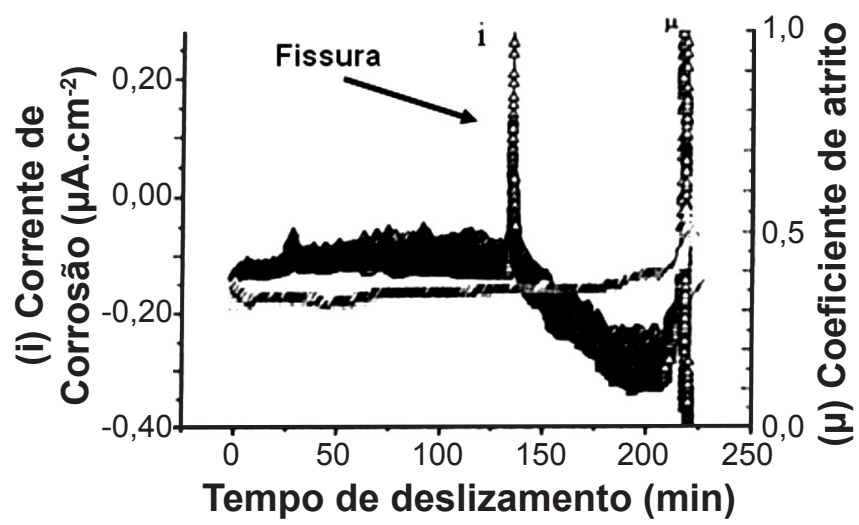

Figura 2: Corrente de corrosão e coeficiente de atrito em função do tempo de deslizamento para a amostra CP2A. Análise detalhada em torno de $\mathrm{i}_{\text {corr }}=0$.

[Figure 2: Corrosion current and coefficient of friction as a function of sliding time for sample CP2A. Detailed analysis around $\left.i_{\text {corr }}=0.\right]$ é muito provável que este ponto corresponda à formação de uma fissura na amostra seguida de eventual repassivação da superfície metálica, correspondente à redução dos valores de corrente.

A micrografia da borda contígua à área desgastada pelo ensaio de tribocorrosão do corpo de prova (Fig. 3) mostra diversas linhas assimétricas de fratura na porcelana. A interface apresenta-se descontínua, sugerindo falhas na união do sistema metalocerâmico após a submissão a ciclos repetitivos de deslizamento com carga em ambiente corrosivo.

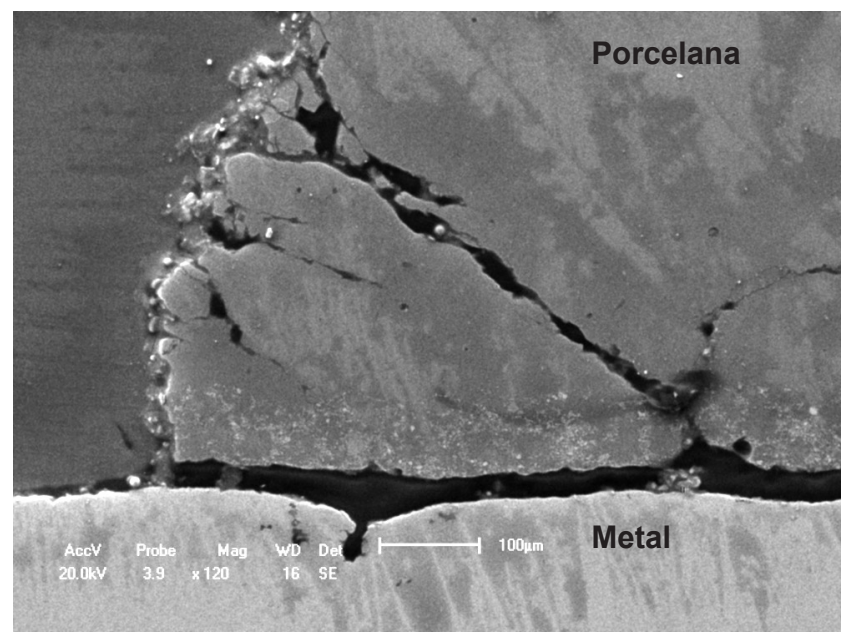

Figura 3: Imagem por MEV da borda contínua à área desgastada pelo ensaio tribocorrosivo de amostra sem filme de titânio.

[Figure 3: SEM image of the border next to the area tested by tribocorrosion of sample with no Ti film.]

A presença de trincas mesmo sem a fratura completa da união é potencialmente prejudicial à estabilidade químicomecânica do sistema metalocerâmico. Quimicamente, a aproximação da saliva com o substrato metálico, facilita as trocas eletrolíticas e a iniciação do fenômeno corrosivo. Uma vez instalado, o processo de corrosão torna-se

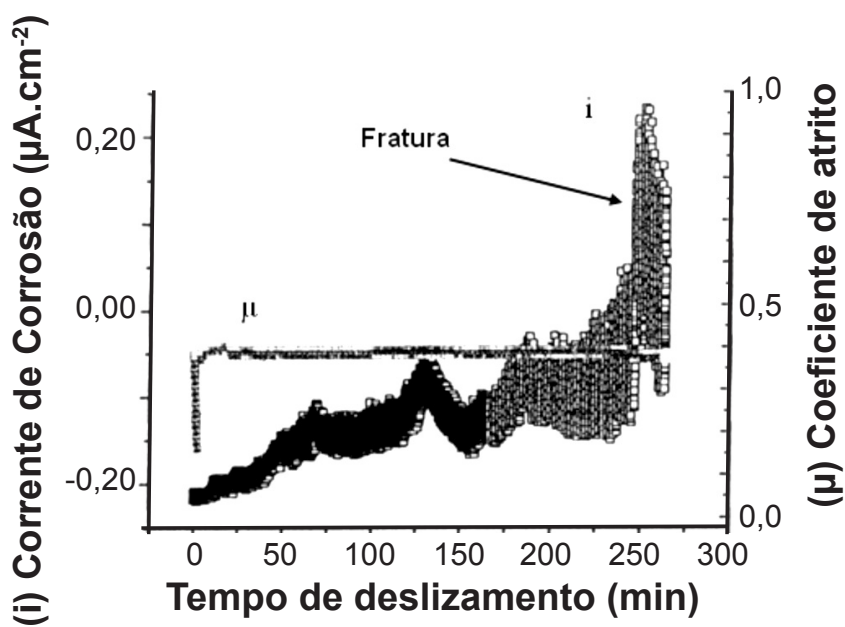

Figura 4: Comportamento tribocorrosivo de amostra do grupo CPB.

[Figure 4: Tribocorrosive behavior of CPB sample.] 


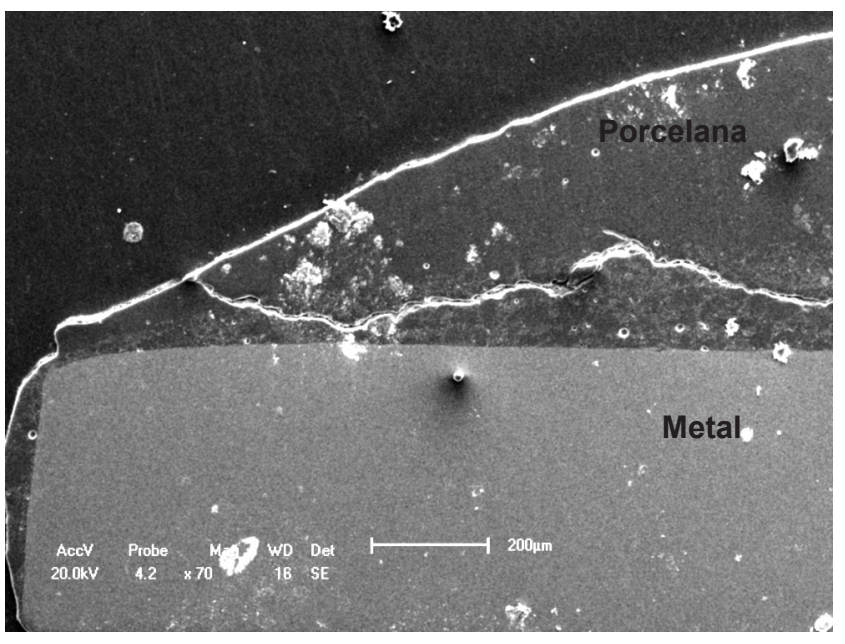

Figura 5: Micrografia eletrônica de varredura da extremidade livre de amostra do grupo CPB.

[Figure 5: SEM image of the border of CPB sample.]

irreversível, sendo particularmente danoso aos pacientes portadores de sensibilidade aos componentes da liga. Do ponto de vista mecânico, a presença de trincas favorece a redução da resistência mecânica do sistema metalocerâmico às solicitações da mastigação. A descontinuidade do componente cerâmico desestabiliza a dissipação da carga mastigatória ao longo da peça protética, atuando como área concentradora de tensão e favorecendo à propagação da trinca.

As amostras metalizadas com titânio demonstraram maior capacidade de repassivação, correspondente à formação de $\mathrm{TiO}_{2}$. Este fato pode ser observado no resultado do ensaio de amostra do grupo CPB (Fig. 4), jateada e metalizada mecanicamente com titânio. À medida que o tempo de deslizamento aumenta após o período de rodagem, a corrente apresenta variações sem respectivo aumento

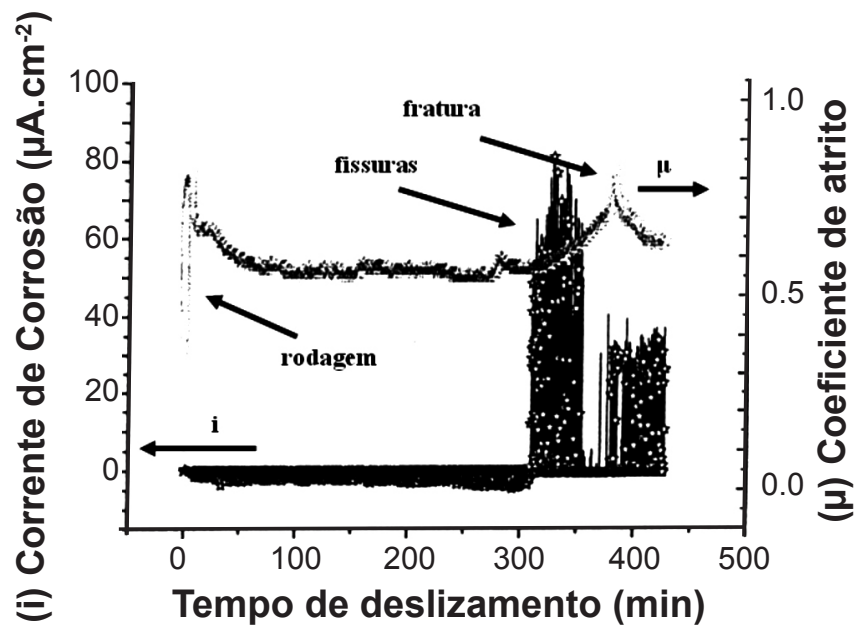

Figura 6: Comportamento tribocorrosivo de amostra do grupo CE. Liga Ni-Cr revestida com titânio depositado em triodo magnetron sputtering.

[Figure 6: Tribocorrosive behavior of CE sample. Ni-Cr alloy coated by triode magnetron sputtering.] do coeficiente de atrito até aproximadamente $250 \mathrm{~min}$ de ensaio, quando ocorre a fratura da amostra. A micrografia da extremidade livre de corpo de prova do grupo CPB (Fig. 5) ilustra um traço extenso de fratura localizado bem acima da interface e que percorre maior área na porcelana de opaco que na porcelana de corpo.

Amostra metalizada com titânio por deposição em plasma (grupo CE) também foi submetida a ensaio tribocorrosivo (Fig. 6). A corrente manteve-se constante e próxima de zero por mais de $300 \mathrm{~min}$. A partir desse ponto, a amostra apresentou fissuras, demonstradas pelo aumento dos valores de corrente, no entanto sem concomitante aumento do coeficiente de atrito. A fratura da amostra parece ter ocorrido após aproximadamente $390 \mathrm{~min}$ de ensaio, correspondendo ao maior tempo de sobrevivência das amostras ensaiadas neste estudo. Este comportamento pode ser atribuído à presença de uma camada fina, porém contínua de titânio na interface metalocerâmica, contribuindo tanto para a passivação do sistema quanto para a maior aderência da interface.

Finalmente, a Fig. 7 ilustra uma micrografia eletrônica da extremidade livre de corpo de prova do grupo $\mathrm{CE}$, revelando linha de fratura expandindo-se da interface à superfície da porcelana, porém sem desgarramento da porcelana mais superficial. A presença de um filme contínuo de titânio nesta área oferece uma barreira física protetora do componente metálico contra a ação corrosiva da saliva.

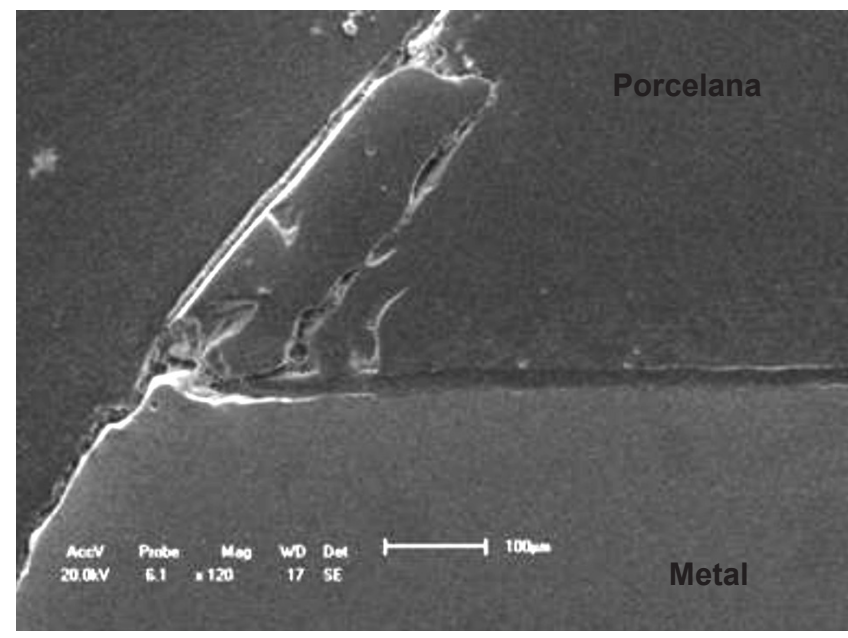

Figura 7: Micrografias eletrônicas de varredura de amostra do grupo CE após ensaio tribocorrosivo.

[Figure 7: SEM images of CE sample after tribocorrosive test.]

\section{CONCLUSÕES}

Este estudo revelou que os métodos de deposição de filmes de titânio mecanicamente ou por plasma têm bom potencial para serem empregados na confecção de próteses fixas metalocerâmicas feitas com ligas à base de níquel-cromo. Relacionado à durabilidade dos sistemas modificados, quando submetidos às cargas cisalhantes em ambiente de saliva artificial, os grupos modificados 
pela presença de intercamada de titânio apresentaram resultados melhores que o grupo sem titânio, tornandose mais favorável para aplicações clínicas que o sistema metalocerâmico convencional. O filme de titânio depositado sobre o substrato de níquel-cromo promove a proteção de interfaces metalocerâmicas contra a agressividade do ambiente corrosivo oral, em decorrência da formação da estável camada de dióxido de titânio. A deposição de titânio por plasma gera filmes uniformes, contínuos e de espessura controlada, enquanto que a deposição mecânica gera filmes não uniformes, descontínuos e de espessura variável. O sistema metal-cerâmica modificado com titânio depositado por triodo magnetron sputtering destacou-se pelo bom comportamento tribocorrosivo, com tempos mais longos de ensaio e picos de repassivação durante o ensaio de tribocorrosão.

\section{AGRADECIMENTOS}

À CAPES e ao CNPq pelo apoio financeiro, e ao Prof. Dr. Luis César Fontana, da Universidade do Estado de Santa Catarina, pelas metalizações em plasma, colaborando fundamentalmente com o estudo.

\section{REFERÊNCIAS}

[1] R. T. Basting, B. Carlini Jr., M. C. Serra, A. L. F. Pimenta, Como Procedimentos Restauradores Diretos Podem Melhorar o Sorriso? Revista Brasileira de Odontologia online, Disponível em < http://www.aborj.org.br $>$, acesso em 16/08/2003.

[2] H. Shillingburg Jr., S. Hobo, L. D. Whitsett, R. Jacobi, S. E. Brackett, Fundamentos de Prótese Fixa, $3^{\text {a }}$ Ed. (1998). [3] G. Isgrò, C. J. Kleverlann, H. Wang, A. J.Feilzer, Thermal dimensional behavior of dental ceramics, Biomaterials $\mathbf{2 5}$ (2004) 2447-2453.

[4] S. Oruç, Y. Tumunoglu, Fit of titanium and base metal alloy metal-ceramic crown, J. Prosthet Dent 83 (2000) 314318.

[5] M.A.Ameer, E. Khamis, M. Al-Motlak, Electrochemical behavior of recasting $\mathrm{Ni}-\mathrm{Cr}$ and $\mathrm{Co}-\mathrm{Cr}$ non-precious dental alloys, Corrosion Sci. 46 (2004) 2825-2836.

[6] O. L. Bezzon, R. F. Ribeiro, J. M. D. A. Rollo, S. Crosara, Castability and resistance of ceramometal bonding in $\mathrm{Ni}-\mathrm{Cr}$ and Ni-Cr-Be alloys, J. Prosthet Dent 85 (2001) 299-304.

[7] H. H. Huang, M. C. Lin, T. H. Lee, H. W. Yang, F. I. Chen, S. C. Wu, C. C. Hsu, Effect of chemical composition of $\mathrm{Ni}$ Cr dental castings alloys on the bonding characterization between porcelain and metal, J. Oral Rehabilitation 32 (2005) 206-212.

[8] M. D. Roach, J. T. Wolan, D. E. Parsell, J. D. Bumgardner, Use of X-ray photoelectron spectroscopy and cyclic polarization to evaluate the corrosion behavior of six nickel-chromium alloys before and after porcelain-fused-to- metal firing, J. Prosthet Dent 84 (2000) 623-634.

[9] W. C. Lee, Strength of $\mathrm{Si}_{3} \mathrm{~N}_{4} \mathrm{Ni}-\mathrm{Cr}$-Fe alloy joints with test methods: shear, three-point and four-point bending, J. Mater. Sci. 32 (1997) 6657-6660.

[10] J. M. D. A. Rollo, S. M. Rossitti, Metal-ceramic restoration: comparative thermal compatibility of $\mathrm{Ni}-\mathrm{Cr}$ alloys and porcelains, Rev. Odontol Univ. S. Paulo 13 (1999) 61-66.

[11] J. Fischer, Ceramic bonding to a dental gold-titanium alloy, Biomaterials 23 (2002) 1303-1311.

[12] C. Ku, S. Park, H. Yang, Comparison of the strengths of metal-ceramic crowns and three ceromer crowns, J. Prosthet Dent 88 (2002) 170-175.

[13] A.D. Bassanta, D. S. Bassanta, Prótese fixa-atualidades e perspectivas, SP, Brasil, Sarvier (1997).

[14] T. M. Hofstede, C. Ercoli, G. N. Graser, R. H. Tallents, M. E. Moss, D. T. Zero, Influence of metal surface finishing on porcelain porosity and beam failure loads at the metalceramic interface, J. Prosthet Dent 84 (2000) 309-317.

[15] D. F. Galindo, C. Ercoli, G. N. Graser, R. H. Tallents, M. E. Moss, Effect of soldering on metal-porcelain bond strength in repairs porcelain-fused-to-metal castings, J. Prosthet Dent 85 (2001) 88-94.

[16] S. F. C. Dekon, L. F. Vieira, G. Bonfante, Avaliação da resistência de união metalocerâmica em função de diferentes tempos de oxidação prévia, Rev Odontol Univ. S. Paulo 13 (1999) 57-60.

[17] C. Hegedus, L. Daróczi, V. Kökényesi, D. L. Beke, Comparative microstructural study of the diffusion zone between $\mathrm{NiCr}$ alloy and different dental ceramics, J. Dent Res. 81 (2002) 334-337.

[18] R. G. Craig, J. M. Powers, Restorative dental materials, $11^{a}$ Ed. Mosby: St Louis (2002).

[19] A. S. Lopez, J. M. Rosca, E. Vasilescu, P. Drob, D. Raducanu, L. Angelescu, Technical and functional properties of some biocompatible thin films, Mater. Chem. Phys. 86 (2004) 38-43.

[20] G. Mabilleau, S. Bourdon, M. L. J. Guillou, R. Filmon, M. F. Baslé, D. Chappard, Influence of fluoride, hydrogen peroxide and lactic acid on corrosion resistance of commercially pure titanium, Acta Biomaterialia 2 (2006) 121-129.

[21] L. C. Fontana, J. C. Sagás, Relatório final de iniciação científica do projeto Deposição de filmes $C N_{x}$ por triodo magnetron sputtering: estudo comparativo de elétrons em um sistema magnetron sputtering em função da configuração e intensidade do campo magnético, Univ. do Estado de Santa Catarina-UDESC (2006).

[22] L. C. Fontana, J. L. R. Muzart, Characteristics of triode magnetron sputtering: the morphology of deposited titanium films, Surface Coatings Technology 107 (1998) 24-30.

[23] P. Ponthiaux, F. Wenger, D. Drees, J. P. Celis, Electrochemical techniques for studying tribocorrosion process, Wear 256 (2004) 459-468.

(Rec. 30/06/2011, Ac. 05/10/2011) 